\title{
Understanding Structure-Function Questions and Crystallization Behavior of Eukaryotic Membrane Proteins by Combining Microscopies
}

\author{
M.C. Johnson*, S. Mari, F. Rudolph*, G. Zhao*, Winfried Haase**, Y. Kanaoka***, D. \\ Stafford ${ }^{* * *}$, D. Müller***** and I. Schmidt-Krey* \\ * Georgia Institute of Technology, School of Biology, School of Chemistry and Biochemistry, \\ Atlanta, GA 30332, U.S.A. \\ **Max-Planck-Institute of Biophysics, Structural Biology, 60439 Frankfurt, Germany. \\ ***Harvard Medical School and Division of Rheumatology, Immunology and Allergy, Brigham and \\ Women's Hospital, Boston, MA 02115, U.S.A. \\ ****The University of North Carolina at Chapel Hill, Dept. of Biology and Center for Thrombosis \\ and Hemostasis, Chapel Hill, NC 27599, U.S.A. \\ ***** Center of Biotechnology, Technical University Dresden, 01307 Dresden, Germany.
}

A detailed understanding of the structure and function of eukaryotic membrane proteins is essential to the treatment and prevention of a large number of diseases as membrane proteins comprise the majority of known and potential drug targets. Structural studies of membrane proteins have presented tremendous difficulties compared to soluble proteins though. The protein needs to be removed from the lipid bilayer and stabilized by detergent, rendering the membrane protein highly sensitive during crystallization. X-ray crystallography has made critical contributions to the still minimal but increasing number of membrane protein structures solved. Important alternative techniques are single particle analysis [1] and electron crystallography of two-dimensional (2D) crystals [2]. Recent methods development in electron crystallography includes critical advances in equipment for controlled, large-scale crystallization, high-throughput screening for $2 \mathrm{D}$ crystals, and image processing [3-7]. The $2 \mathrm{D}$ crystallization process most commonly involves reconstitution of the purified and solubilized membrane protein into a phospholipid bilayer. During this process the detergent is usually removed completely. This results in both an optimal membrane environment for the protein as well as the elimination of the additional factor of a detergent contributing to the complexity of the crystallization. The ensuing 2D crystals in the form of proteoliposomes reveal structural information not only by electron crystallography, but can yield further data by atomic force microscopy (AFM) and freeze-fracture. Additionally, 2D crystallization trials can yield important data for 3D crystallization trials as well as for combining electron and X-ray crystallography studies for structure-function studies.

Over-expressed and purified human leukotriene $\mathrm{C}_{4}$ synthase $\left(\mathrm{LTC}_{4} \mathrm{~S}\right)$ and human vitamin $\mathrm{K}$ dependent $\gamma$-glutamyl carboxylase (GGCX) were induced to form 2D crystals by detergent removal through dialysis for 4 and 8-14 days, respectively $[8,9]$. These preparations were screened for ordered arrays in negative stain with a JEM-1400 transmission electron microscope equipped with Orius and Ultrascan CCD cameras as well as PASyS crystal searching software [4]. Once 2D crystals had formed successfully, additional 2D crystallization parameters were tested to increase size and quality of crystals as well as to examine conditions that might lead to generally applicable 2D crystallization screens in the long-term. Two-dimensional crystals were then prepared for freezefracture, high-resolution data collection at 4K with a JEOL 3000 SFF electron microscope, and AFM. Tightly packed proteoliposomes were also used for freeze-fracture and AFM. 
The combination of microscopies allowed us to gain structural information on transmembrane $\alpha$ helices and the oligomeric arrangement as well as further insights into the 2D crystallization process. It is hoped that findings on 2D crystal formation will eventually lead to an increase in the rate of $2 \mathrm{D}$ crystallization due to a more in-depth understanding of how and why 2D crystals form, as well as how the formation of higher quality crystals can be induced. It is expected that with additional data these studies will contribute to overcoming the bottleneck in electron crystallography by providing detailed general 2D crystallization guidelines, and demonstrating the importance of combining techniques to understand the structure and function of many more membrane proteins. Surprisingly, the number of $\mathrm{LTC}_{4} \mathrm{~S}$ subunits is not limited to the trimer that had previously been observed by both electron and X-ray crystallography $[8,11,12]$. Structure-function studies are underway to understand the functional implications of both the trimeric and the larger oligomeric organization of human $\mathrm{LTC}_{4} \mathrm{~S}$. Furthermore, the studies of these eukaryotic membrane proteins as well as data from trials of other membrane proteins have profited from applying a range of microscopies, namely TEM, AFM, and freeze-fracture with further benefits to 3D crystallization.

\section{References}

[1] M. Radermacher, Methods in Enzymology, Mitochondrial Function. Ed. W.S. Allison \& I.E. Scheffler, (2009) Vol. 456, 3.

[2] I. Schmidt-Krey, Methods 41 (2007) 417.

[3] A. Cheng et al., J. Struct. Biol. 160 (2007) 324.

[4] N. Nakamura et al., Microsc. Microanal. 9 (suppl. 2) (2003) 1038.

[5] M. Vink et al., J. Struct. Biol. 160 (2007) 295.

[6] A. Engel et al., Proc. of the 46th Annual Meeting of the Electron Microsc. Soc. of America (1988) 152.

[7] B. Gipson et al., J. Struct. Biol. 160 (2007) 475.

[8] Schmidt-Krey et al., Structure 12 (2004) 2009.

[9] Schmidt-Krey et al., J. Struct. Biol. 157 (2007) 437.

[10] Zhao et al., J. Struct. Biol. 169 (2010) 450.

[11] Ago et al., Nature (2007) 448, 609-612.

[12] Martinez Molina et al., Nature (2007) 448, 613-616.

[13] This research is supported by NIH Grant HL090630. 